\title{
Dose Rate Measurements in Pulsed Radiation Fields by Means of an Organic Scintillator
}

\author{
Theresa Werner ${ }^{1}$, Roland Beyer ${ }^{2}$, Richard Biedermann ${ }^{1}$, Marko Gerber ${ }^{1}$, Jürgen Götze ${ }^{3}$, Philipp Herzig ${ }^{1}$, \\ Vincent Melzer $^{1}$, Elena Metzner ${ }^{1}$, David Weinberger ${ }^{2,4}$ and Thomas Kormoll ${ }^{1,4}$ \\ ${ }^{1}$ Institue of Nuclear and Particle Physics, Technische Universität Dresden, Germany \\ ${ }^{2}$ Helmholtz-Zentrum Dresden-Rossendorf, Germany \\ ${ }^{3}$ Helios Klinikum Aue, Germany \\ ${ }^{4}$ Serious Dynamics, Germany \\ theresa.werner@tu-dresden.de
}

\begin{abstract}
A deficiency in the implementation of current radiation protection is the determination of the ambient dose equivalent $H^{*}(10)$ and the directional dose equivalent $H^{\prime}(0.07)$ in pulsed radiation fields. Conventional dosimeter systems are not suitable for measurements in photon fields comprising short radiation pulses, which consequently leads to high detector loads in short time periods. Nevertheless, due to the implementation of advanced medical accelerators for cancer therapy, new medical diagnostic devices as well as various laser machining systems, there is an urgent need for suitable dosimeter systems for real time dosimetry. In this paper, a detector concept based on an organic scintillator and a full digital data analysis with the aim of developing a portable, battery powered measurement system is presented.
\end{abstract}

Keywords - radiation protection, dose rate measurements, pulsed radiation fields, organic scintillation detector

\section{INTRODUCTION}

$\mathrm{M}$ any dosimetric measurement systems are not suitable for the application in pulsed radiation fields regarding radiation protection scenarios. Especially in applications where a low mean dose rate in the range of $1 \mu \mathrm{Sv} / \mathrm{h}$ is applied. A main challenge in such fields is to process high detector loads within short time periods, while an appropriate dead time behavior and the suppression of pile up effects must be ensured. A promising approach for an active dosimetric system fulfilling these requirements is the combination of a fast tissue equivalent scintillation detector coupled to a full digital signal processing unit. Such a system could allow real time dosimetry by measuring the deposited energy in the detector, while a discrimination between pulsed and non-pulsed events is realized by comparing the individual time stamps of the measured events. Additionally, pile up events can be identified by analyzing the pulse shape of the individual events.

Furthermore, due to the completely non-paralyzed dead time behavior of the detection system, it is possible to correct signal losses for the respective measurement. The principle of this method, which is in detail described in [1], was implemented in an appropriate detection system based on an organic scintillator and a digital data acquisition board. The developed prototype was tested in a continuous as well as pulsed photon fields.

In a measurement campaign at the institute of nuclear and particle physics (IKTP) of Technische Universität Dresden, Germany, with a ${ }^{137} \mathrm{Cs}$ source, quantitative dose rate measurements were performed. To determine the dose values an appropriate analyzing algorithm for dose rate measurements was developed.

At the $\gamma$ ELBE bremsstrahlung-facility [2] a pulsed photon beam was available, where measurements under various pulse frequencies (up to $10 \mathrm{kHz}$ ) and a macro pulse duration of $40 \mu \mathrm{s}$ were performed. The detection system was placed next to a polymethylmethacrylat (PMMA) phantom, which was irradiated with the bremsstrahlung-beam.

Additionally, dose rate measurements at a clinical TrueBeam therapeutic system by Varian [3] were performed, where the detector was placed outside the treatment room.

For both measurements in pulsed radiation fields, it was possible to reconstruct the characteristic structure of the pulsed beam, which comprises the identification of the pulse length and repetition rate.

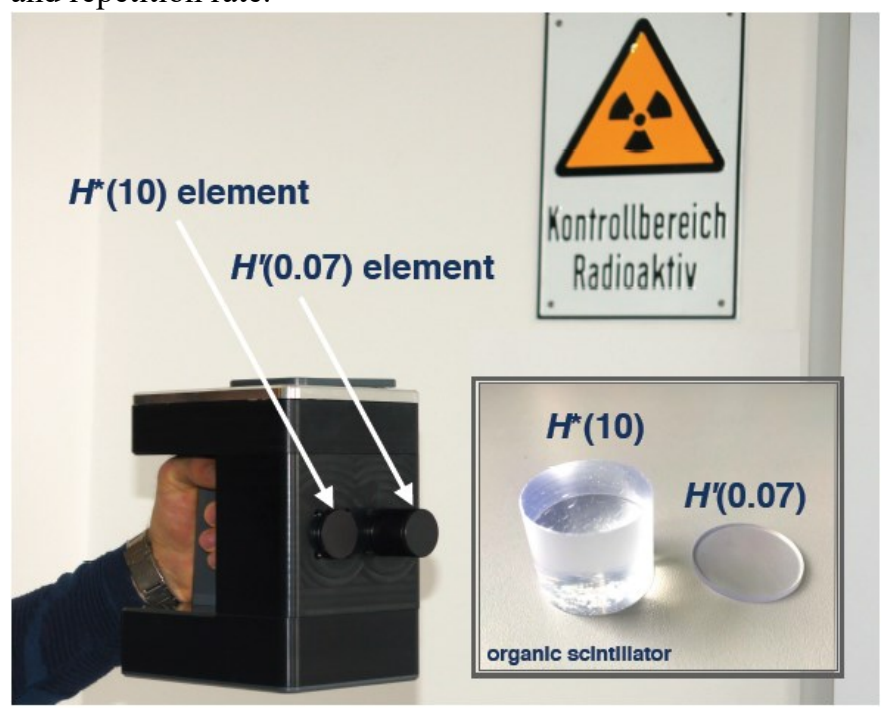

Figure 1. The detection system is a portable, battery powered device with two organic scintillation elements $\left(H^{*}(10)\right.$ element, and $H^{\prime}(0.07)$ element) each coupled on an individual photomultiplier tube with a subsequent free running 14 bit analog-to-digital converter with a sampling rate of $125 \mathrm{MHz}$ for data 


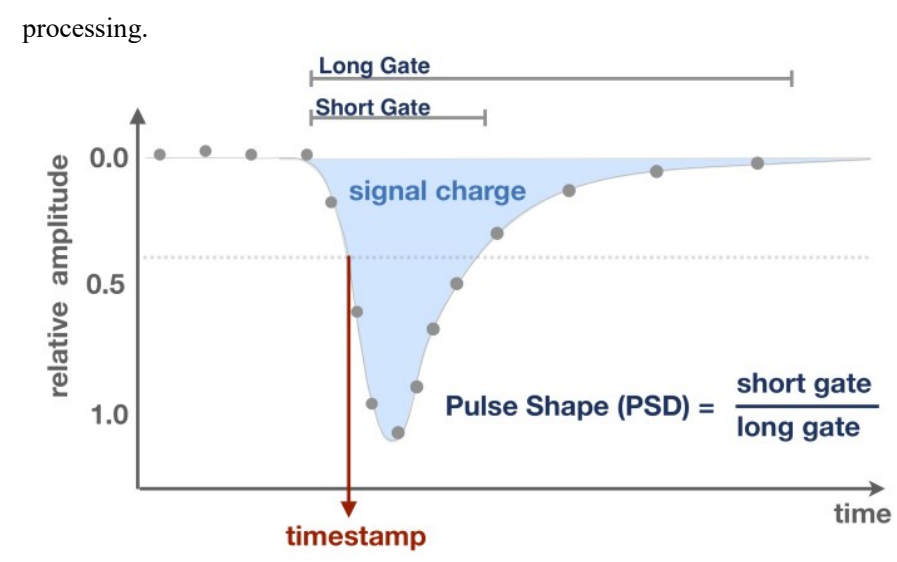

Figure 2. The voltage pulses coming from the two photomultiplier tubes are digitized by an analogue-to-digital converter (125 MHz, 14 Bit) and processed by a field programmable gate array (FPGA). In the sketch the three individual analysis parameter are shown: (1) the timestamp, which determines the arrival time of the detected event, (2) the pulse charge, which is the integral over the signal and proportional to the deposited energy and (3) the pulse shape parameter (PSD), which represents the shape of the signal.

\section{METHODS AND MATERIAL}

A photograph of the detection system is shown in Figure 1. It consists of two detection elements $\left(H^{*}(10)\right.$ element, $H^{\prime}(0.07)$ element) for the respective radiation protection quantities. The cylindrical organic scintillators have a height of $19 \mathrm{~mm}$ and a diameter of $23 \mathrm{~mm}$ for measuring $H^{*}(10)$ and a height of $1 \mathrm{~mm}$ and a diameter of $24 \mathrm{~mm}$ for measuring $H^{\prime}(0.07)$. Each scintillator is coupled to an individual photomultiplier tube (PMT: PDM 04-9111) produced by ETEnterprises, which are read out with a free running 14 bit analogue-to-digital converter (ADC). For this, the output signal is sampled with a data acquisition board DAQ125 by Serious Dynamics, Dresden, Germany, which allows a real-time processing of the data via an included field programmable gate array (FPGA).

\section{A. Data processing}

As shown in the sketch of Figure 2, for each recorded event signal the FPGA algorithm determines three distinct parameters: (1) The first one is the timestamp, which represents the pulse onset with respect to the number of elapsed clock cycles. (2) The second parameter is the pulse charge, which is defined by the integral over the whole pulse (long gate). (3) The third is the pulse shape parameter (PSD), which is given by the ratio of the partly integrated pulse (front part of the signal / short gate) to the integral of the whole pulse. This parameter is therefore a measure for the shape of the pulse and thus allows to distinguish between different "event scenarios" in the scintillation detector (e.g. single event, pile-up event, particle discrimination).

\section{B. Data analysis}

As introduced in section 1, a main challenge for a dose compliance measurement in pulsed radiation fields is to handle the high detector load within short time periods $(\mathrm{Hz}-\mathrm{kHz})$ and at the same time short pulse durations (down to ns - fs). In the detected data the dead time behavior of the detection system as well as the probability of pile-up events must be considered.

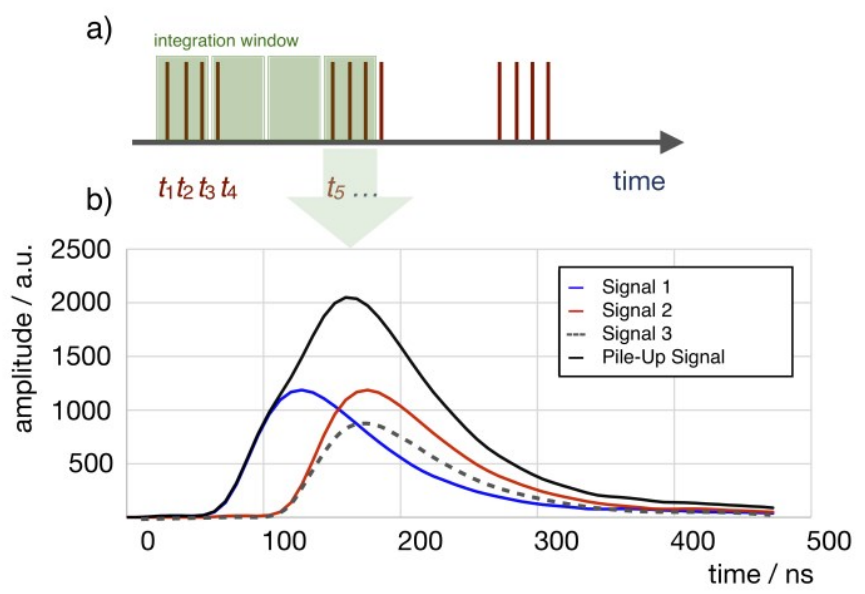

Figure 3.The residual signal of multiple events (Pile-Up Signal), which are detected in the same integration window (event 1: signal 1, event 2: signal 2), is proportional to the deposited energy in the detector (tissue equivalent) and therefore to the ambient and directional dose. This also applied to for different energy depositions (signal 3) due to the equal weighting factor.

Because of the mentioned FPGA real-time processing the detection system shows a non-paralysable dead time behavior due to the subsequent integration gates (Figure 3a).

The system dead time is thereby defined by the length of the integration window (long gate for determining the pulse charge). If multiple events occur in the same integration window the resulting multi-event-signal (Pile-up signal) comprises the deposited energies of each detected single event (Figure 3b). As the organic scintillation material is approximately tissue equivalent, the weighting factor of different energy deposition (Figure 3b: signal 1 and signal 3 (dashed line)) is the same as for equal energy deposition (Figure 3b: signal 1 and signal 2).

\section{Experimental Setups}

In the following, the experimental setups of the three performed experiments are described. In section a) the measurement in a continuous photon field is characterized, while section b) and c) comprise two experiments in different pulsed photon fields.

a) Radioactive nuclide (continuous): At the IKTP an experiment was performed in a continuous radiation field of a ${ }^{137}$ Cs source (Figure 4). Within this experiment, it was intended to proof the implemented algorithm by measuring the dose and dose rate under defined conditions. The measured values at different distances to the source were therefore compared to the data from an ionization chamber UNIDOS webline [4] by Physikalisch-Technische Werkstätten (PTW) Freiburg.

b) Research accelerator (pulsed): In a further experiment at the $\gamma$ ELBE bremsstrahlung-facility the detector was exposed to a scattered photon field, in which the time structure of the initial beam was received. The micro-frequency of $13 \mathrm{MHz}$ is not regarded in this study and assumed to be quasi continuous at the studied time scales. The accelerator was operated to produce a 


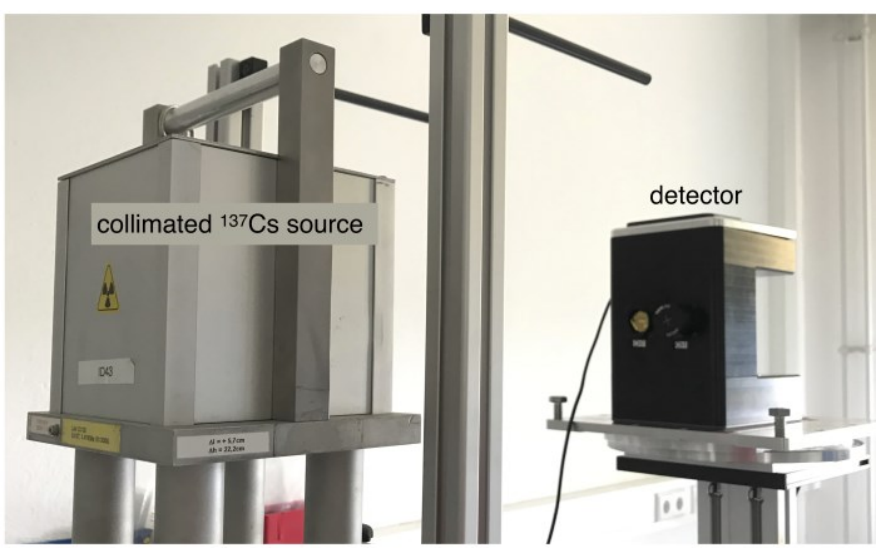

Figure 4. The developed detection system was irradiated with a collimated ${ }^{137} \mathrm{Cs}$ source to determine quantitative dose/dose rate values.

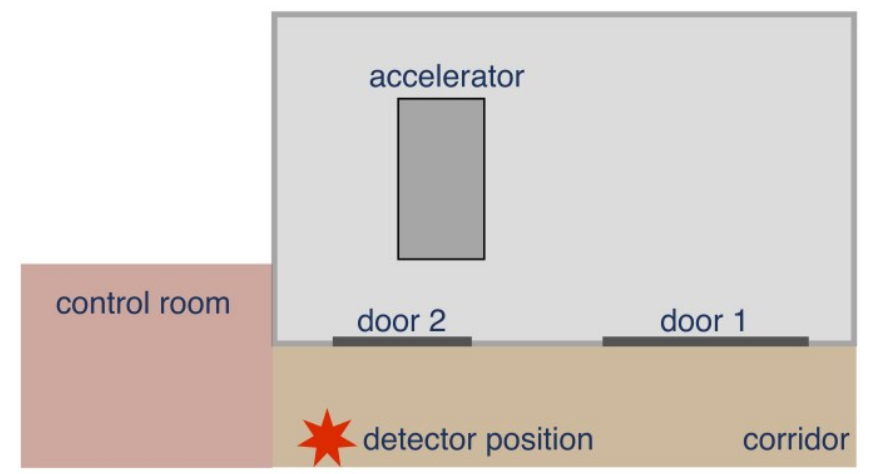

Figure 5. At the Helios Klinikum, Aue, Germany, a measurement next to the control room was performed, while at the same time an experiment was carried out inside the treatment room.

continuous wave (cw) as well as a pulsed bremsstrahlung-beam with a respective pulse frequency of $5 \mathrm{kHz}$ and $10 \mathrm{kHz}$. The pulse duration was set to $40 \mu \mathrm{s}$.

c) Medical accelerator (pulsed): At the Helios Klinikum Aue, Germany, a TrueBeam therapeutic system by Varian produces a pulsed photon beam, where the frequency depends on the requested dose / dose rate in the treatment and can therefore vary. Here, the detector was placed outside the treatment room in the adjacent corridor behind a door to the treatment room (Figure 5). Inside the treatment room a PMMA target was irradiated with a $30 \mathrm{~cm} \times 30 \mathrm{~cm}$ field and a $15 \mathrm{MV}$ photon beam. The dose rate in the treatment room was set to $5 \mathrm{~Gy} / \mathrm{min}$ for the performed experiment.

\section{RESULTS AND DISCUSSION}

\section{A. Radioactive Source: ${ }^{137} \mathrm{Cs}$}

Figure 6 shows the pulse charge spectra for the different detection elements (red: $H^{*}(10)$ element, gray: $H^{\prime}(0.07)$ element) measured in the radiation field of a ${ }^{137} \mathrm{Cs}$ source. As the scintillation material is a low $Z$-scintillator $\left(Z_{\text {eff }} \sim 4.5[5]\right)$ the characteristic ${ }^{137} \mathrm{Cs}$ peak at $662 \mathrm{keV}$ is not visible in the respective spectra. To calibrate the detectors, the Compton edges (c.f. Figure 6) and dosimetric values from the data of the

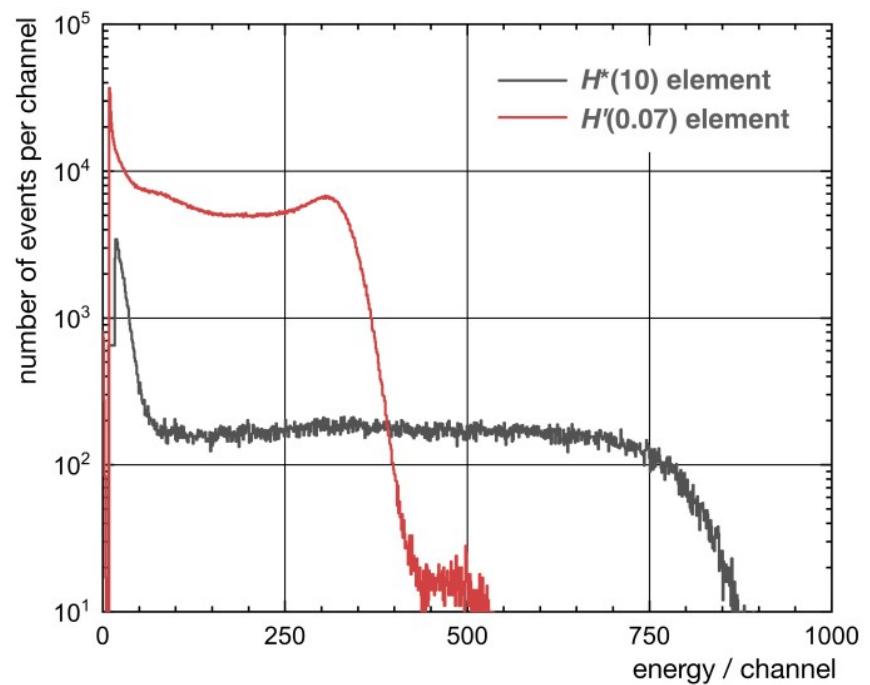

Figure 6 . The pulse-charge histograms for the two individual detector elements (gray: $H^{*}(10)$ element, red: $H^{\prime}(0.07)$ element) measured during the irradiation with a ${ }^{137} \mathrm{Cs}$ source. The Compton edges are clearly visible.

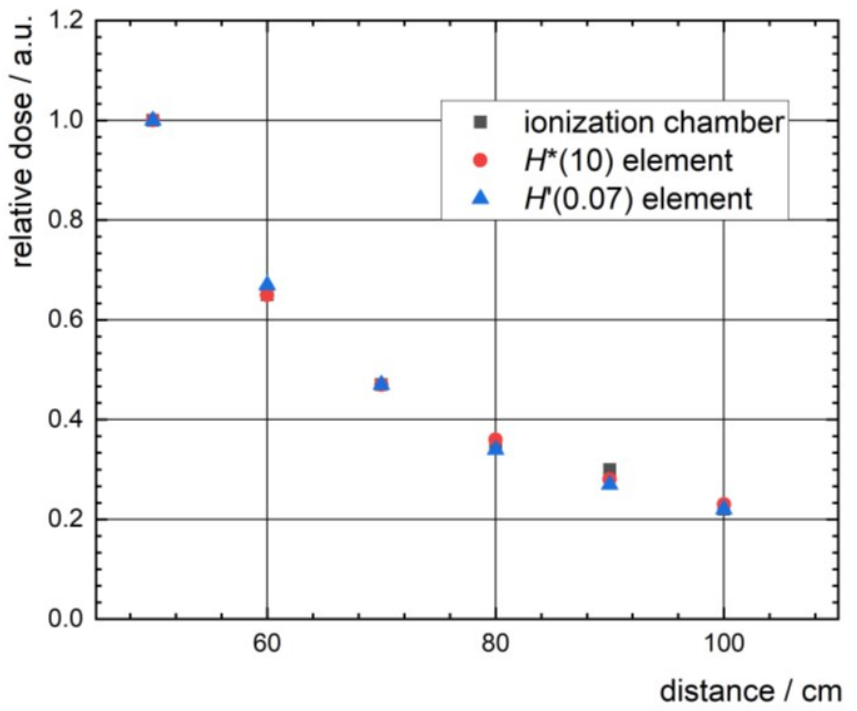

Figure 7. The relative dose decreases with greater distances. The black values belong to the reference measurement with an ionization chamber (UNIDOS webline), the red to the $H^{*}(10)$ element and the blue to the $H^{\prime}(0.07)$ element of the detection system. All measured values are normalized to the respective first value recorded at a distance of $50 \mathrm{~cm}$.

UNIDOS webline ionization chamber were used. For this, a reference measurement at a fixed position was performed. This, together with the pulse charge spectra (Compton edge), allowed an energy calibration and therefore a comparison of dose and dose rate values between the detection system and the ionization chamber. The measured relative dose values at the different positions are given in Figure 7.

Here, the values were normalized to the respective first value at a distance of $d=50 \mathrm{~cm}$. Consequently, at this position the 


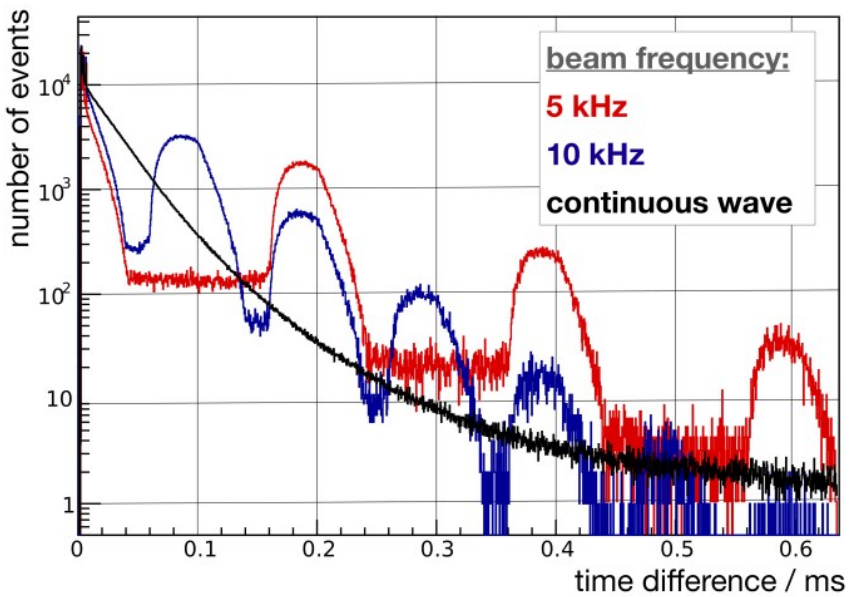

Figure 8. In the time-difference histogram, the timing structure of the initial beam is reproducible. The black curve belongs to the continuous wave beam and the red and blue curve to a pulsed beam with a pulse duration of $40 \mu$ s and a frequency of $5 \mathrm{kHz}$ and $10 \mathrm{kHz}$, respectively.

relative dose for the different detectors is equal to 1 . For the different detector elements the exposed dose decreases, as expected, with greater distances and the single values match with the reference measurement (ionization chamber). This proofs that the developed detection system is suitable to evaluate dose as well as dose rate quantities in continuous radiation photon fields.

\section{B. Research Accelerator: ELBE}

To reconstruct the timing structure of the primary beam the difference between two subsequent measured events was calculated. The resulting time-difference histogram is shown in Figure 8. The red curve represents the measurement of a pulsed beam with a frequency of $5 \mathrm{kHz}$ and the blue one with a frequency of $10 \mathrm{kHz}$. The black curve belongs to the $\mathrm{cw}$ measurement. In these histograms the time structure of the initial beam can be clearly identified, where the periodic peak structure gives the respective beam period $(0.2 \mathrm{~ms}$ and $0.1 \mathrm{~ms})$ (consequently, the above mentioned frequencies) and the peak widths encode the beam pulse duration. In contrast to the pulsed beam, the time differences for events, which are measured during a continuous beam, follow a falling monotonous distribution.

\section{Medical Accelerator: TrueBeam}

Similar to the above described experiment, the time-difference histogram (Figure 9) was measured outside the treatment room during the operation of a clinical accelerator. The determined frequency of the accelerator was reconstructed from the measured data with around $166 \mathrm{~Hz}$ for the specific beam setting.

\section{SUMMARY AND OUTLOOK}

Based on the performed experiments, it could be shown that the timing structure of various pulsed radiation photon fields can be reconstructed out of the recorded data. This allows, for

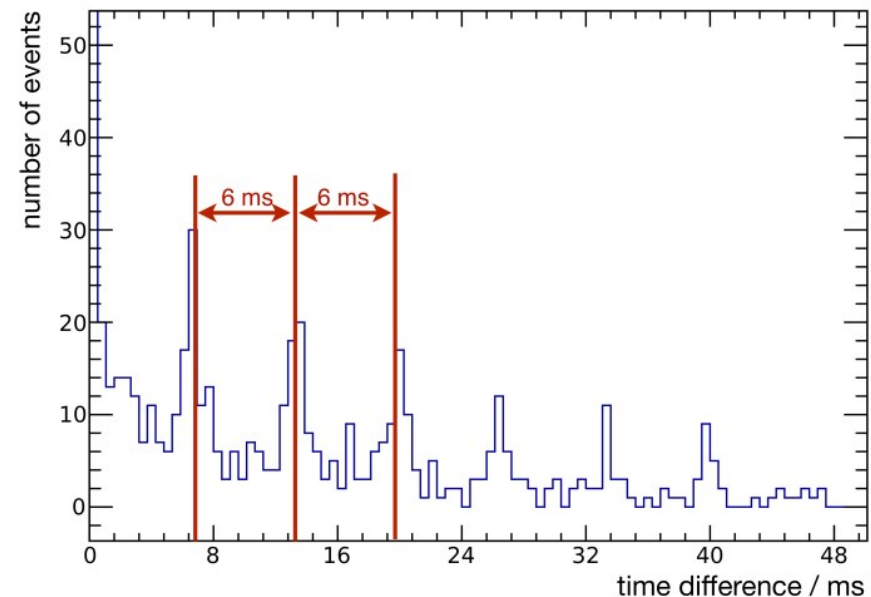

Figure 9. The time-difference histogram measured in front of the treatment room at Helios Klinikum, Aue, Germany, during the operation of a clinical accelerator. In the recorded data, the time structure of the initial beam could be reconstructed with a frequency of around $166 \mathrm{~Hz}$.

example, the discrimination between detected events, which are correlated to the pulsed beam, and possible continuous background radiation in future experiments. Furthermore, this enables a correction of the dose and dose rate values for applications under different conditions (mixed photon fields). The first quantitative measurements in a continuous photon field proof that the system is in principle suitable for the evaluation of dose and dose rate values. In future experiments, this will be additionally tested in pulsed photon fields.

The detections system will be also tested in low energy photon fields $(\sim \mathrm{keV})$ to ensure an appropriate calculation of the radiation protection quantities for low doses and dose rates.

For this, an accurate energy calibration is needed, which will be obtained from a further experiment based on a coincidence scattering technique. The detailed method is described in reference [6] and [7].

\section{REFERENCES}

[1] K. Makarevich et al., "Dosimetry with the ability to distinguish pulsed and non-pulsed dose contributions", Radiation Protection Dosimetry, 2020 .

[2] R. Schwengner et al., "The photon-scattering facility at the superconducting electron accelerator ELBE”, Nucl. Instr. Meth., 2005.

[3] C- Glide-Hurst et al., "Commissioning of the Varian TrueBeam linear accelerator: a multi-insitutional study“, Med Phys. 2013.

[4] PTW Freiburg, "Grebrauchsanweisung 30 $\mathrm{cm}^{3}$ Stielkammer Typ 23361", operating instruction, 2001.

[5] G. F. Knoll, ,Radiation detection and measurement”, Wiley $4^{\text {th }}$ ed. 2010.

[6] Swiderski et al., "Measurement of Compton edge position in low-Z scintillators", Radiation Measurements, 2010.

[7] V. Melzer, "Realisierung eines roboter-unterstützten WeitwinkelCompton-Aufbaus zur Energiekalibrierung von Niedrig-ZSzintillatoren“, bachelor thesis, 2020. 